\title{
BL Lacertae Objects and the Extragalactic $\gamma$-Ray Background
}

\author{
Fan $\mathrm{LI}^{1,2}$ and Xinwu $\mathrm{CAO}^{1}$ \\ ${ }^{1}$ Key Laboratory for Research in Galaxies and Cosmology, Shanghai Astronomical Observatory, \\ Chinese Academy of Sciences, 80 Nandan Road, Shanghai, 200030, China \\ ${ }^{2}$ Graduate School of the Chinese Academy of Sciences, Beijing 100039, China \\ lifan@shao.ac.cn
}

(Received ; accepted )

\begin{abstract}
A tight correlation between $\gamma$-ray and radio emission is found for a sample of BL Lacertae (BL Lac) objects detected by Fermi Gamma-ray Space Telescope (Fermi) and the Energetic Gamma-Ray Experiment Telescope (EGRET). The $\gamma$-ray emission of BL Lac objects exhibits strong variability, and the detection rate of $\gamma$-ray BL Lac objects is low, which may be related to the $\gamma$-ray duty cycle of BL Lac objects. We estimate the $\gamma$-ray duty cycle, $\delta_{\gamma} \simeq 0.11$, for BL Lac objects detected by EGRET and Fermi. Using the empirical relation of $\gamma$-ray emission with radio emission and the estimated $\gamma$-ray duty cycle $\delta_{\gamma}$, we derive the $\gamma$-ray luminosity function (LF) of BL Lac objects from their radio LF. Our derived $\gamma$-ray LF of BL Lac objects can almost reproduce that calculated with the recently released Fermi bright active galactic nuclei (AGN) sample. We find that $\sim 45 \%$ of the extragalactic diffuse $\gamma$-ray background (EGRB) is contributed by BL Lac objects. Combining the estimate of the quasar contribution to the EGRB in the previous work, we find that $\sim 77 \%$ of the EGRB is contributed by BL Lac objects and radio quasars.
\end{abstract}

Key words: galaxies: active - galaxies: BL Lacertae objects: general — cosmology: diffuse radiation — gamma rays: theory

\section{Introduction}

There are 66 high-confidence identifications of blazars in the third catalog of active galactic nuclei (AGN) detected by the Energetic Gamma-Ray Experiment Telescope (EGRET) on the Compton Gamma-Ray Observatory (CGRO), which include 20 BL Lac objects (e.g. Hartman et al. 1999; Mattox et al. 2001). The LAT bright AGN Sample (LBAS) detected by Fermi includes 104 blazars consisting of 42 BL Lac objects, 57 flat spectrum radio quasars 
(FSRQs), and 5 blazars with uncertain classification (Abdo et al. 2009a). Comptonization is believed to be responsible for the $\gamma$-ray emission from blazars, which can be classified into two categories: the external Comptonization (EC) model and the synchrotron self-Comptonization (SSC) model, according to the origin of the soft seed photons (see, e.g., Böttcher 2007 for a review and references therein ). In the EC models, the soft seed photons are assumed to originate from the accretion disks, the broad-line regions (BLRs), or/and the dust tori (e.g., Ghisellini \& Madau 1996; Georganopoulos et al. 2001; Dermer \& Schlickeiser 2002). It was suggested that the SSC model may be responsible for $\gamma$-ray radiation from BL Lac objects while the EC model predominates over SSC model for quasars (e.g., Dondi \& Ghisellini 1995). The statistical analysis on a sample of EGRET blazars implied that the soft seed photons may be predominantly from the BLRs (e.g., Fan \& Cao 2004; Fan, Cao \& Gu 2006), which is consistent with the lack of BLR and other external fields emission in most BL Lac objects. Their results are roughly consistent with the detailed modeling of SEDs for a large sample of $\gamma$-ray bright AGNs by (Ghisellini et al. 2010), in which all external field photons from BLR, accretion disk, and dust torus, are properly considered. However, the situation becomes more complicated for radiation in other wavebands (e.g., see Kataoka \& Stawarz 2005; Bai et al. 2009; Liu et al. 2008). It was found that the X-ray radiation from some FSRQs (e.g., 3C 273) is consistent with combined SSC and EC mechanisms (e.g., Pacciani et al. 2009).

The extragalactic diffuse $\gamma$-ray background (EGRB) was first discovered by the SAS 2 satellite (Fichtel et al. 1978; Thompson \& Fichtel 1982) and subsequently confirmed after the launch of EGRET (Michelson 1995; Sreekumar et al. 1998). The EGRB integrated above 100 $\mathrm{MeV}$ was determined to be $(1.45 \pm 0.05) \times 10^{-5}$ photons $\mathrm{cm}^{-2} \mathrm{~s}^{-1} \mathrm{sr}^{-1}$ (Sreekumar et al. 1998). Using a new model of the Galactic background, Strong et al. (2004) obtained a slightly smaller value of the EGRB, $(1.14 \pm 0.12) \times 10^{-5}$ photons $\mathrm{cm}^{-2} \mathrm{~s}^{-1} \mathrm{sr}^{-1}$. Almost all extragalactic $\gamma$-ray sources in the third EGRET catalog are identified as blazars, which account for about $13 \%$ of the EGRB (Hartman et al. 1999). The contribution of unresolved blazars to the EGRB has been explored in many previous works, either by extrapolating the observed $\gamma$-ray luminosity distribution to obtain a $\gamma$-ray luminosity function (LF) or using the correlation of $\gamma$-ray emission with the emission in radio bands derived from EGRET blazars, which showed that about $\sim 25 \%$ to $\sim 100 \%$ of the EGRB can be attributed to the unresolved blazars (e.g., Padovani et al. 1993; Chiang et al. 1995; Stecker \& Salamon 1996; Chiang \& Mukherjee 1998; Mücke \& Pohl 2000; Narumoto \& Totani 2006; Cao \& Bai 2008).

A new revised catalogue of EGRET $\gamma$-ray sources (EGR2008) was given by Casandjian \& Grenier (2008), and a larger catalogue of the LAT Bright AGN Sample (LBAS) detected by Fermi was released recently (Abdo et al. 2009a). In this paper, we derive a correlation between $\gamma$-ray and radio emission for a sample of BL Lac objects detected by EGRET or/and Fermi (Casandjian \& Grenier 2008; Abdo et al. 2009a). We estimate the $\gamma$-ray duty cycle of BL Lac objects detected by EGRET and Fermi, and the $\gamma$-ray LF of BL Lac objects is calculated 


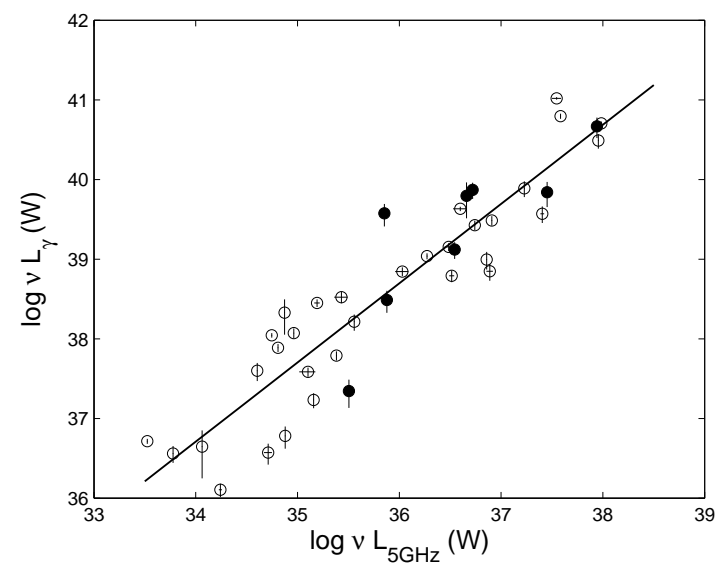

Fig. 1. The relation of $K$-corrected radio luminosity and $\gamma$-ray luminosity for a sample of $\gamma$-ray BL Lac objects detected by Fermi/EGRET. The solid line represents the linear regression for all the sources in the sample. Open circles: Fermi BL Lac objects; filled circles: EGRET BL Lac objects.

with this empirical correlation from their radio LF and $\gamma$-ray duty cycle. The radio LF of BL Lac objects derived by Padovani et al. (2007) based on the Deep X-ray Radio Blazar Survey (DXRBS) is used in this work. We further estimate the total number of BL Lac objects to be detected by Fermi, and the contribution of all BL Lac objects to the EGRB. The cosmological parameters $\Omega_{\mathrm{M}}=0.3, \Omega_{\Lambda}=0.7$, and $H_{0}=70 \mathrm{~km} \mathrm{~s}^{-1} \mathrm{Mpc}^{-1}$ have been adopted in this paper.

\section{The correlation between $\gamma$-ray and radio emission}

The recently released LAT Bright AGN Sample (LBAS) detected by Fermi includes 42 BL Lac objects, of which 11 sources were already detected by EGRET. Till now, there are 51 BL Lac objects detected either by EGRET or Fermi [with the Test Statistic (TS) value above the EGRET threshold ( $4 \sigma$ for $|b|>10^{\circ}$, and $5 \sigma$ for $|b|<10^{\circ}$ ); or $10 \sigma$ for the sources detected by Fermi]. There are 40 BL Lac objects with measured redshifts, which are adopted for our present investigation. The radio/ $\gamma$-ray flux and photon index $\Gamma$ above $100 \mathrm{MeV}$ for the sources are listed in Table 1. In figure 1, we plot the relation between radio luminosity $L_{\mathrm{R}, 5 \mathrm{G}}$ at $5 \mathrm{GHz}$ and $\gamma$-ray luminosity $L_{\gamma}(\geq 100 \mathrm{MeV})$ for these BL Lac objects, where the average K-corrected radio and $\gamma$-ray fluxes are adopted for calculating $L_{\gamma}$ and $L_{\mathrm{R}, 5 \mathrm{G}}$. We find a significant correlation between $L_{\mathrm{R}, 5 \mathrm{G}}$ and $L_{\gamma}$ (the correlation coefficient $r=0.926$ ). The linear regression of the sample gives

$$
\log \nu L_{\gamma}=0.995 \log \nu L_{\mathrm{R}, 5 \mathrm{G}}+2.886 .
$$

Abdo et al. (2009a) investigated the relation between $8.4 \mathrm{GHz}$ radio flux density and $\gamma$-ray flux density for Fermi blazar sample. The peak values are adopted by Abdo et al. (2009a), which are likely to correspond to a short time-scale flare state of a source. Kovalev et al. (2009) investigated the relation between quasi-simultaneous $\gamma$-ray and $15 \mathrm{GHz}$ radio flux densities in Fermi blazar sample, which includes xx BL Lac objects. The mean fluxes above $100 \mathrm{MeV}$ 


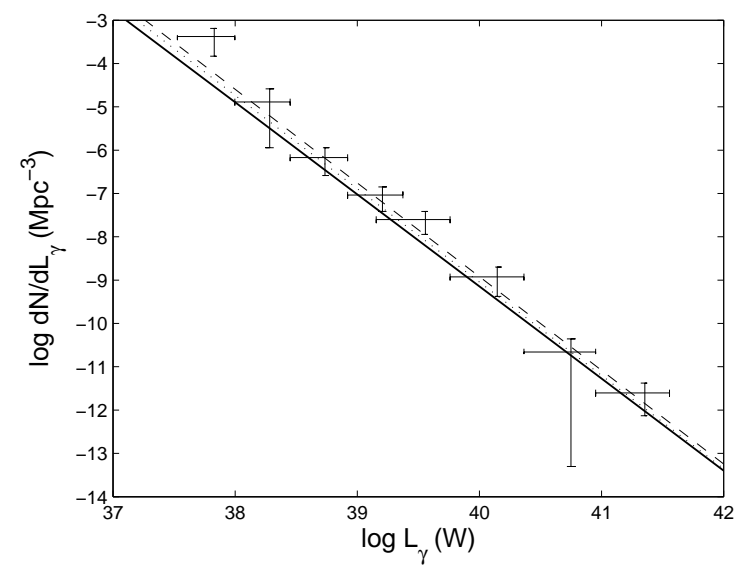

Fig. 2. The $\gamma$-ray LFs for BL Lac objects. The solid line is the LF derived in this work, while the dashed line and the dotted line are the LFs (redshift less than 0.3 and greater than 0.3 ) calculated with the LAT bright AGN sample detected by Fermi (Abdo et al. 2009a).

are adopted in this work, and the sample is limited to BL lac objects, which should be a suitable choice, because we focus on the statistic properties of $\gamma$-ray BL Lac objects as a whole population in this work. The significance of the correlation found in this work is higher than that found by Abdo et al. (2009a), which may be attributed to the fact that only the peak $\gamma$-ray fluxes were adopted in their analysis. For $\gamma$-ray quasars, the situation is more complicated, as their $\gamma$-ray emission is not only dependent on their radio emission, but also on the origins of the soft seed photons, which is beyond the scope of this work.

\section{The $\gamma$-ray luminosity function of BL Lac objects}

Based on the correlation between $L_{\mathrm{R}, 5 \mathrm{G}}$ and $L_{\gamma}$ established with a sample of BL Lac objects, we can calculate the $\gamma$-ray LF of BL Lac objects from their radio LF. Recently, Padovani et al. (2007) derived a LF for BL Lac objects based on the Deep X-ray Radio Blazar Survey (DXRBS), which extends to lower luminosity than that derived by Stickel et al. (1991). In this work, we use Padovani et al. (2007)'s radio LF to derive the $\gamma$-ray LF of BL Lac objects.

Although a tight correlation between $L_{\mathrm{R}, 5 \mathrm{G}}$ and $L_{\gamma}$ is found for $\gamma$-ray BL Lac objects, we find that only a small fraction of BL Lac objects were detected by EGRET, which may imply that the detection of blazars in $\gamma$-ray energy band is not only dependent on their radio emission, but also on their $\gamma$-ray activity. The $\gamma$-ray emission is closely related with radio emission for BL lac objects based on SSC models. More distant or/and fainter BL Lac objects can be detected if the sensitivity is improved. Abdo et al. (2009a) found that the redshift distribution of Fermi BL Lac objects $(z \leq 1.2)$ is similar to that of the DXRBS BL Lac sample $(z \lesssim 1.0)$ (Padovani et al. 2007). This may imply that the radio-gamma correlation found in this work has not been affected by the redshift distribution of $\gamma$-ray BL Lac objects. Thus, 
we induce a $\gamma$-ray duty cycle $\delta_{\gamma}$ for BL Lac objects, which can be estimated from the ratio of $\gamma$-ray BL Lac objects to the total (e.g., Stecker \& Salamon 1996; Vercellone et al. 2004). There are 36 BL Lac objects were detected by EGRET and Fermi, among which the lowest radio flux density at $5 \mathrm{GHz}$ is $\simeq 0.25 \mathrm{Jy}$. The number of BL Lac objects above this flux density limit with the same sky coverage of the survey carried out with EGRET/Fermi is 330 (e.g., see figure 4 in Padovani et al. 2007), which leads to $\delta_{\gamma} \simeq 0.11$. Vercellone et al. (2004) estimate the $\gamma$-ray activity using three different methods. Our result is consistent with the maximum distribution of blazars active fraction computed in Vercellone et al. (2004) (see Fig.14 of Vercellone et al. (2004)).

The $\gamma$-ray LF of BL Lac objects can then be calculated with

$$
\Phi_{\gamma}\left(L_{\gamma}, z\right)=\delta_{\gamma} \Phi_{\mathrm{R}}\left(L_{\mathrm{R}, 5 \mathrm{G}}, z\right) \frac{\mathrm{d} L_{\mathrm{R}, 5 \mathrm{G}}}{d L_{\gamma}}=\frac{\delta_{\gamma} L_{\mathrm{R}, 5 \mathrm{G}}}{0.995 L_{\gamma}} \Phi_{\mathrm{R}}\left(L_{\mathrm{R}, 5 \mathrm{G}}, z\right)
$$

where $\Phi_{\gamma}$ is the $\gamma$-ray LF, and the radio LF $\Phi_{R}$ is given by Padovani et al. (2007). We can use a simple GLF model defined as

$$
\Phi_{\gamma}\left(L_{\gamma}, z\right) \propto L_{\gamma}^{\beta}
$$

In figure 2 , we compared our $\gamma$-ray LF $(\beta=-2.13)$ derived from the radio LF and that directly derived with a sample of $\gamma$-ray BL Lac objects detected by Fermi (Abdo et al. 2009a). The slope of our $\gamma$-ray LF is well in agreement with the value of $-2.17 \pm 0.05$ reported for Fermi BL Lac objects. Our $\gamma$-ray LF is slightly less than that of (Abdo et al. 2009a), but taking into account the observational error, we can also say our $\gamma$-ray LF is roughly consistent with that of Fermi.

The number count of BL Lac objects as a function of $\gamma$-ray flux above $100 \mathrm{MeV}$ can be calculated with the derived $\gamma$-ray LF,

$$
N\left(\geq f_{\nu, \gamma}^{\min }\right)=\int_{0}^{z_{\mathrm{m}}} \frac{d V}{d z} d z \int_{4 \pi d_{\mathrm{L}}^{2} f_{\nu, \gamma}^{\min }} \phi_{\gamma}\left(L_{\nu, \gamma}, z\right) d L_{\nu, \gamma}
$$

where $z_{\mathrm{m}}=1$ is adopted in our calculations, because the radio LF for BL Lac objects was derived with a sample of sources with redshifts $z=0-1$ (see Padovani et al. 2007, for the details). This means that the results derived here are only the lower limits. In fact, almost all $\gamma$-ray BL Lac objects detected have redshifts $z \lesssim 1$ (only 2 of 40 have redshifts slightly higher than unity). We plot the number count of $\gamma$-ray BL Lac objects derived from the LF calculated with equation (2) in figure 3. Obviously, the increasing number of sources can be detected following a decreasing flux limit, such as $N \sim 500$ at $f_{\text {limit }}=0.06 f_{\text {limit }}^{E G R E T}$ (the Fermi sensitivity for one year) (Gehrels \& Michelson 1999), and $N \sim 1000$ at $f_{\text {limit }}=1 / 30 f_{\text {limit }}^{E G R E T}$ (the Fermi sensitivity for two year) (Atwood et al. 2009).

The EGRB contributed by all BL Lac objects can be calculated with the $\gamma$-ray LF derived with equation (2), 


$$
f_{\mathrm{EGRB}}=\frac{1}{4 \pi} \int_{0}^{z_{\mathrm{m}}} d z \int_{4 \pi d_{\mathrm{L}}^{2} f_{\nu, \gamma}^{\min }} \frac{d N\left(L_{\nu, \gamma}, z\right)}{d z d L_{\nu, \gamma}} \frac{L_{\nu, \gamma}(1+z)^{2-\Gamma}}{4 \pi d_{\mathrm{L}}^{2} E_{100 \mathrm{MeV}}} d L_{\nu, \gamma},
$$

where the average photon spectral index $\Gamma=2.04$ for BL Lac objects is adopted, which is slightly higher than $\Gamma=1.99 \pm 0.22$ given by Abdo et al. (2009a). This is due to that our sample includes 9 EGRET sources, of which the photon spectral indexes are all greater than 2.0 except $1011+496$. The contribution of the BL Lac objects to the EGRB as a function of $\gamma$-ray flux limit $f_{\text {limit }}$ is plotted in figure 4 .

\section{Discussion}

It was suggested that the $\gamma$-ray radiative mechanisms are different for quasars and BL Lac objects, i.e., the EC mechanism may be responsible for quasars, while the SSC is for BL Lac objects (e.g., Dondi \& Ghisellini 1995). A linear relation between radio and $\gamma$-ray emission is expected for AGNs, if the SSC mechanism is responsible for $\gamma$-ray emission (see, e.g., equation (28) of Dermer et al. 1997). The correlation between radio and $\gamma$-ray emission for BL Lac objects found in this work is very close to a linear one as described in equation(1), which seems to support the SSC mechanism for $\gamma$-ray emission from BL Lac objects.

The variability of $\gamma$-ray emission in 10 sources of 42 Fermi BL Lac objects have been detected. The faction is much less than that of FSRQs (45/57)(Abdo et al. 2009a), which implies that the variability of $\gamma$-ray emission from BL Lac objects could be weaker than that from FSRQs. The detection rate in $\gamma$-ray band may be dominantly related to the duty cycle of $\gamma$-ray BL Lac objects. Vercellone et al. (2004) investigated the $\gamma$-ray activity of EGRET blazars and estimated their $\gamma$-ray duty cycle. They found that about 48 percent of the sources fall into $\delta_{\gamma}=0-5 \%$, and 74 percent sources are in $\delta_{\gamma} \leq 10 \%$. The duty cycle $\delta_{\gamma} \simeq 0.11$ derived

in this work is roughly consistent with the results in Vercellone et al. (2004) (while their $\gamma$-ray duty-cycle was computed without a clear distinction between BL Lacs and FSRQs.).

In this work, the $\gamma$-ray LF of BL Lac objects is derived from the radio LF by using the empirical correlation of $\gamma$-ray emission with radio emission, which can almost reproduce the $\gamma$-ray LF calculated directly with the $\gamma$-ray BL Lac objects detected by Fermi/EGRET (see figure 2). Based on the derived $\gamma$-ray LF for BL Lac objects, we can calculate the number count of $\gamma$-ray BL Lac objects as a function of flux limit in $\gamma$-ray band. We find that about 1000 BL Lac objects will be detected by Fermi, if its sensitivity is 30 times higher than that of EGRET at $100 \mathrm{MeV}$ (Gehrels \& Michelson 1999). Cao \& Bai (2008)'s estimate show that about 1200 quasars can be detected by Fermi based on the EC mechanism for their $\gamma$-ray emission. The recently released bright AGN sample detected by Fermi together with the sources already detected by EGRET with high-confidence leads to a sample of $135 \gamma$-ray blazars (77 quasars, 51 BL Lac objects and 7 blazars with uncertain classification). The ratio of quasars to BL Lac objects for the present sample (LBAS+EGRET) is about 1.5, which is slightly higher than 


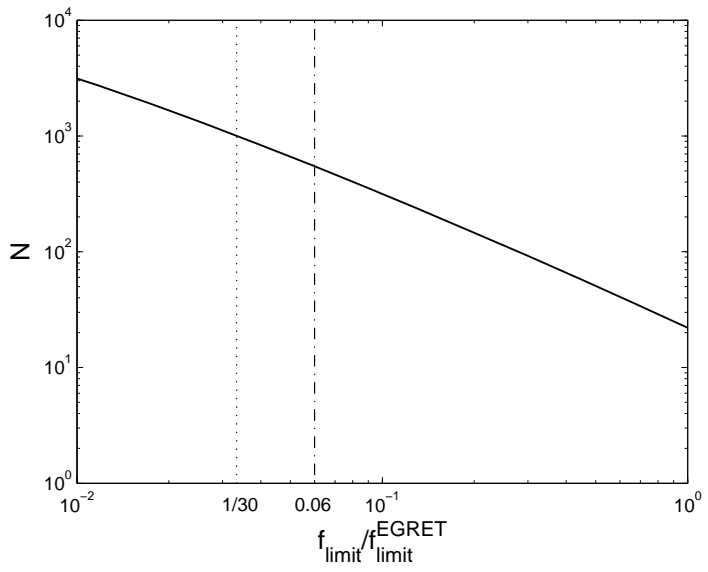

Fig. 3. The number count of $\gamma$-ray BL Lac objects as a function of flux limit at $100 \mathrm{MeV}$. The dotted line and dot-dashed line represents the sensitivity of Fermi. We note $f_{\min } \simeq 5 \times 10^{-8} \mathrm{ph} \mathrm{cm}^{-2} \mathrm{~s}^{-1}$ for EGRET (see, e.g., Cao \& Bai 2008). The flux limit of Fermi at $100 \mathrm{MeV}$ can be 30 times lower than that of EGRET for a two year all-sky survey(Gehrels \& Michelson 1999) and the sensitivity for one year will

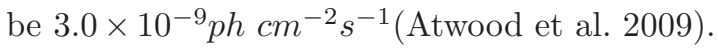

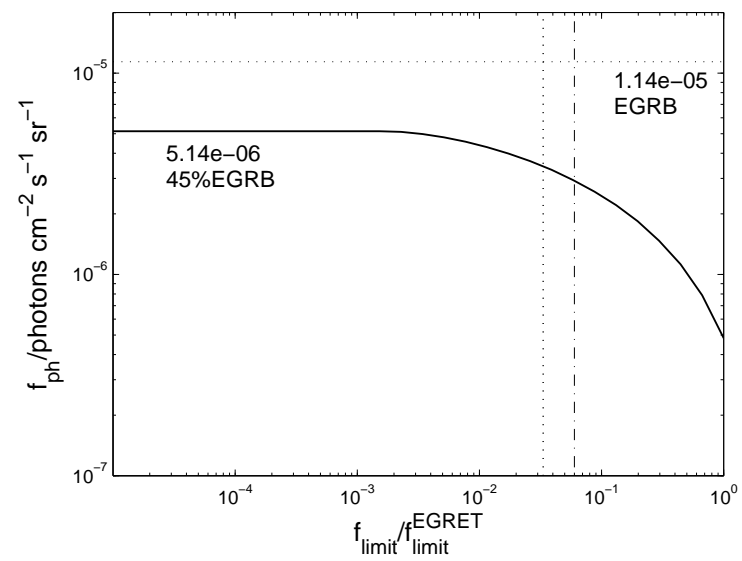

Fig. 4. The contribution of the BL Lac objects with $\gamma$-ray flux $f \geq f_{\text {limit }}$. The dashed line indicates the measured EGRB with photon energy above $100 \mathrm{MeV}$. We use the value of the EGRB obtained by Strong et al. (2004), $(1.14 \pm 0.12) \times 10^{-5}$ photons $\mathrm{cm}^{-2} \mathrm{~s}^{-1} \mathrm{sr}^{-1}$. The symbols of dotted line and dot-dashed line as in Figure 3. 
1200/1000, which implies that more BL Lac objects are expected to be detected by the Fermi in the future.

Integrating the derived $\gamma$-ray LF, we calculate the contribution of all BL Lac objects to the EGRB, which accounts for $\sim 45 \%$ of the EGRB (see figure 4). Cao \& Bai (2008)'s calculation show that all radio quasars and FR II radio galaxies contribute about $\sim 32 \%$ of the EGRB. This means that the contribution of BL Lac objects is similar to that of quasars, which is roughly consistent with the predicted number ratio of quasars to BL Lac objects to be detected by Fermi. The contribution of all blazars (quasars+BL Lac objects) can account for $\sim 77 \%$ of the EGRB, which implies there still some space $(\lesssim 23 \%)$ is left for other sources to the EGRB. Considering that our calculations are limited to $z=0 \sim 1$ for BL Lac objects, the contribution of the sources other than blazars to the EGRB should be lower than the value derived in this work. We also noticed the detection of $\gamma$-ray emission from relativistic jets in the narrow-line Seyfert-1 galaxies (PMN 0948+0022)(Abdo et al. 2009b), which means that the mechanism of $\gamma$-ray radiation from narrow-line Seyfert-1 galaxies is similar to that for blazars. Considering that a fraction of narrow-line Seyfert-1 galaxies are radio-loud (e.g. Komossa et al. 2006; Yuan et al. 2008) and some of them show evidence for relativistic jets (e.g. Gu \& Chen 2010), the contribution of radio-loud narrow-line Seyfert-1 galaxies to the EGRB may be important, however, the detailed calculation is beyond the scope of this work.

We thank the referee for his/her helpful comments, and J. M. Bai for helpful discussion. This work is supported by the NSFC (grants 10773020, 10821302 and 10833002), the CAS (grant KJCX2-YWT03), the Science and Technology Commission of Shanghai Municipality (10XD1405000), and the National Basic Research Program of China (grant 2009CB824800). 


\section{References}

Abdo, A. A. et al. 2009a, ApJ, 700, 597A

Abdo, A. A. et al. 2009b, ApJ, 699, 976

Atwood, W. B. et al. 2009ApJ, 697, 1071

Bai, J. M., Liu, H. T., \& Ma, L. 2009, ApJ, 699, 2002

Bloom, S. D., 2007, AIPC, 921, 299

Böttcher, M., 2001, Ap\&SS, 309, 95

Böttcher, M. 2007, Ap\&SS, 309, 9

Cao, X., \& Bai, J. M. 2008, ApJ, 673L, 131

Cara, M. \& Lister, M. L. 2000, BAAS, 39, 784

Casandjian J.-M., Grenier I. A., 2008, A\&A, 489, 849

Chiang, J., Fichtel, C. E., von Montigny, C., Nolan, P. L., \& Petrosian, V. 1995, ApJ, 452, 156

Chiang, J., Mukherjee, R. 1998, ApJ, 496, 752

Dermer, C. D., Sturner, S. J., \& Schlickeiser, R. 1997, ApJS, 109, 103

Dermer, C. D., \& Schlickeiser, R. 2002, ApJ, 575, 667

Dondi, L, \& Ghisellini, G. 1995, MNRAS, 273, 583

Fan, Z.-H., \& Cao, X. 2004, ApJ, 602, 103

Fan, Z.-H., Cao, X., \& Gu, M. 2006, ApJ, 646, 8

Fichtel, C. E., Simpson, G. A., \& Thompson, D. J. 1978, ApJ, 222, 833

Gehrels, N., \& Michelson, P. 1999, Astroparticle Physics, 11, 277

Georganopoulos, M., Kirk, J. G., \& Mastichiadis, A. 2001, ApJ, 561, 111

Ghisellini, G., \& Madau, P. 1996, MNRAS, 280, 67

Ghisellini, G., Tavecchio, F., \& Chiaberg, M. 2005, A\&A, 432, 401

Ghisellini, G., et al. 2010, MNRAS, 402, 497G

Ghosh, K. K. , Ramsey, B. D., \& Sivaram, C. 1999, Bulletin of the American Astronomical Society, 31,665

Gu, M., \& Chen, Y. 2010, arXiv:1004.3058

Hartman, R. C., et al. 1999, ApJS, 123, 79

Kataoka, J., \& Stawarz, Ł. 2005, High Energy Gamma-Ray Astronomy, 745, 522

Komossa, S., Voges, W., Xu, D., Mathur, S., Adorf, H.-M., Lemson, G., Duschl, W. J., \& Grupe, D. 2006, AJ, 132, 531

Kovalev et al. 2009, ApJL, 696,17

Liu, H. T., Bai, J. M., \& Ma, L. 2008, ApJ, 688, 148

Mattox, J. R., Hartman, R. C., \& Reimer, O. 2001, ApJS, 135, 155

Mücke, A., \& Pohl, M. 2000, MNRAS, 312, 177

Michelson, P. F. 1995, grsc, conf, 159

Narumoto, T., \& Totani, T. 2006, ApJ, 643, 81

Pacciani, L., et al. 2009, A\&A, 494, 49

Padovani, P., et al. 1993, MNRAS, 260, L21

Padovani, P., Giommi, P., landt, H., \& Perlman, E. S. 2007, ApJ, 662,182

Salamon, M. H., \& Stecker, F. W. 1994, ApJ, 430, L21 
Sikora, M., Begelman, M. C., \& Rees, M. J. 1994, ApJ, 421, 153

Sikora, M., \& Madejski, G. 2001, American Institute of Physics(AIP), 558, 275

Sreekumar, P., et al. 1998, ApJ, 494, 523

Stecker, F. W., Salamon, M. H., \& Malkan, M. A. 1993, ApJ, 410, L71

Stecker, F. W., \& Salamon, M. H. 1996, ApJ, 464, 600

Stickel, M., Fried, J. W., Kuehr, H., Padovani, P., \& Urry, C. M. 1991, ApJ, 374, 431

Strong, A. W., Moskalenko, I. V., \& Reimer, O. 2004, ApJ, 613, 956

Thompson, D. J., \& Fichtel, C.E. 1982, A\&A, 109, 352

Travis, A. Rector \& John, T.Stocke. 2001, AJ, 122, 565

Vercellone, S., Soldi, S., Chen, A. W., \& Tavani, M. 2004, MNRAS, 353, 890

Yang, J., \& Fan, J. 2005, CJAA, 5, 229

Yuan, W., Zhou, H. Y., Komossa, S., Dong, X. B., Wang, T. G., Lu, H. L., \& Bai, J. M. 2008, ApJ, 685,801 
Table 1. Literature Data for $\gamma$-ray BL Lac objects

\begin{tabular}{|c|c|c|c|c|c|c|c|c|c|}
\hline IAU name & source name & $\mathrm{z}$ & $\begin{array}{r}F_{5 G H z} \\
(\mathrm{Jy})\end{array}$ & $\begin{array}{r}\Delta F_{5 G H z} \\
(\mathrm{Jy})\end{array}$ & ref & $\Gamma$ & $\Delta \Gamma$ & 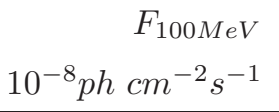 & $\begin{array}{r}\Delta F_{100 \mathrm{MeV}} \\
10^{-8} \mathrm{ph} \mathrm{cm}^{-2} \mathrm{~s}^{-1}\end{array}$ \\
\hline $0048-097$ & 0FGL J0050.5 - 0928 & 0.537 & 1.92 & $\ldots$ & 3 & 2.15 & 0.08 & 10.2 & 1.4 \\
\hline $0109+224$ & 0FGL J0112.1 + 2247 & 0.265 & 0.2575 & 0.0382 & 4 & 2.10 & 0.09 & 7.4 & 1.2 \\
\hline $0118-272$ & 0FGL J0120.5 - 2703 & 0.557 & 1.18 & $\ldots$ & 3 & 1.99 & 0.14 & 3.2 & 0.8 \\
\hline $0219+428$ & 0FGL J0222.6 + 4302 & 0.444 & 0.953 & 0.165 & 4 & 1.97 & 0.04 & 25.9 & 1.6 \\
\hline $0235+164$ & 0FGL J0238.6 + 1636 & 0.940 & 1.48 & 0.15 & 5 & 2.05 & 0.02 & 72.6 & 2.5 \\
\hline $0301-243$ & 0FGL J0303.7 - 2410 & 0.260 & 0.39 & $\ldots$ & 3 & 2.01 & 0.13 & 3.9 & 0.9 \\
\hline $0332-403$ & 0FGL J0334.1 - 4006 & 1.445 & 2.6 & $\ldots$ & 3 & 2.15 & 0.12 & 5.3 & 1.1 \\
\hline $0426-380$ & 0FGL J0428.7 - 3755 & 1.112 & 1.14 & $\ldots$ & 3 & 2.14 & 0.05 & 24.5 & 1.8 \\
\hline $0447-439$ & 0FGL J0449.7 - 4348 & 0.205 & 0.2365 & 0.015 & 6 & 2.01 & 0.06 & 12.0 & 1.3 \\
\hline $0502+675$ & 0FGL J0507.9 + 6739 & 0.416 & 0.025 & $\ldots$ & 4 & 1.67 & 0.18 & 1.7 & 0.8 \\
\hline $0521-365$ & EGR J0529-3608 & 0.055 & 9.23 & $\ldots$ & 3 & 2.63 & 0.42 & 17.57 & 6.8 \\
\hline $0537-441$ & 0FGL J0538.8 - 4403 & 0.892 & 3.8 & $\ldots$ & 3 & 2.19 & 0.04 & 37.6 & 2.2 \\
\hline $0716+714$ & 0FGL J0722.0 + 7120 & 0.310 & 1.12 & 0.01 & 2 & 2.08 & 0.05 & 16.4 & 1.4 \\
\hline $0735+178$ & 0FGL J0738.2 + 1738 & 0.424 & 3.14 & 0.12 & 5 & 2.10 & 0.14 & 4.6 & 1.1 \\
\hline $0814+425$ & 0FGL J0818.3 + 4222 & 0.530 & 0.956 & $\ldots$ & 10 & 2.07 & 0.08 & 9.6 & 1.3 \\
\hline $0829+046$ & EGR J0829+ 0510 & 0.180 & 0.700 & $\ldots$ & 3 & 2.47 & 0.40 & 16.53 & 5.1 \\
\hline $0847-120$ & EGR J0852 - 1224 & 0.566 & 0.834 & 0.045 & 7 & 1.58 & 0.58 & 23.2 & 11.1 \\
\hline $0851+202$ & 0FGL J0855.4 + 2009 & 0.306 & 2.30 & 0.12 & 5 & 2.31 & 0.11 & 9.0 & 1.5 \\
\hline $0954+658$ & EGR J0956 + 6524 & 0.368 & 1.46 & $\ldots$ & 1 & 2.08 & 0.24 & 12.65 & 3.0 \\
\hline $1011+496$ & 0FGL J1015.2 + 4927 & 0.212 & 0.146 & $\ldots$ & 10 & 1.73 & 0.07 & 4.9 & 0.7 \\
\hline $1011+496$ & EGR J1009+ 4831 & 0.200 & 0.146 & $\ldots$ & 10 & 1.90 & 0.37 & 5.63 & 1.7 \\
\hline $1053+494$ & 0FGL J1053.7 + 4926 & 0.140 & 0.0427 & $\ldots$ & 9 & 1.42 & 0.20 & 0.5 & 0.3 \\
\hline $1055+567$ & 0FGL J1058.9 + 5629 & 0.143 & 0.146 & $\ldots$ & 10 & 2.11 & 0.14 & 3.9 & 1.0 \\
\hline $1101+384$ & 0FGL J1104.5 + 3811 & 0.030 & 0.421 & 0.06 & 10 & 1.77 & 0.04 & 15.3 & 1.1 \\
\hline $1215+303$ & 0FGL J1218.0 + 3006 & 0.130 & 0.262 & $\ldots$ & 10 & 1.89 & 0.06 & 9.7 & 1.1 \\
\hline $1219+285$ & 0FGL J1221.7 + 2814 & 0.102 & 0.6755 & 0.17 & 5 & 1.93 & 0.07 & 8.3 & 1.1 \\
\hline $1334-127$ & EGR J1337 - 1310 & 0.539 & 2.18 & $\ldots$ & 3 & 2.62 & 0.42 & 18.7 & 6.5 \\
\hline $1514-241$ & 0FGL J1517.9 - 2423 & 0.048 & 1.907 & 0.17 & 5 & 1.94 & 0.14 & 4.1 & 1.2 \\
\hline $1553+113$ & 0FGL J1555.8 + 1110 & 0.360 & 0.638 & 0.096 & 4 & 1.70 & 0.06 & 8.0 & 1.0 \\
\hline $1604+159$ & EGR J1607 + 1533 & 0.357 & 0.281 & $\ldots$ & 10 & 2.06 & 0.41 & 39.3 & 12.3 \\
\hline $1652+398$ & 0FGL J1653.9 + 3946 & 0.033 & 1.1945 & 0.05 & 5 & 1.70 & 0.09 & 3.1 & 0.6 \\
\hline $1717+178$ & 0FGL J1719.3 + 1746 & 0.137 & 0.94 & $\ldots$ & 3 & 1.84 & 0.07 & 6.9 & 0.9 \\
\hline $1730-130$ & EGR J1734 - 1315 & 0.902 & 4.10 & $\ldots$ & 3 & 2.23 & 0.10 & 32.54 & 9.3 \\
\hline $1749+096$ & 0FGL J1751.5 + 0935 & 0.322 & 1.495 & 0.21 & 5 & 2.27 & 0.07 & 18.4 & 2.1 \\
\hline
\end{tabular}


Table 1. (Continued.)

\begin{tabular}{|c|c|c|c|c|c|c|c|c|c|}
\hline IAU name & source name & $\mathrm{z}$ & $\begin{array}{r}F_{5 G H z} \\
(\mathrm{Jy}) \\
\end{array}$ & $\begin{array}{r}\Delta F_{5 G H z} \\
(\mathrm{Jy}) \\
\end{array}$ & ref & $\Gamma$ & $\Delta \Gamma$ & $\begin{array}{r}F_{100 M e V} \\
10^{-8} \mathrm{ph} \mathrm{cm}^{-2} \mathrm{~s}^{-1} \\
\end{array}$ & 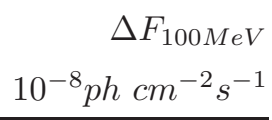 \\
\hline $1803+784$ & 0FGL J1802.2 + 7827 & 0.680 & 2.8 & $\ldots$ & 5 & 2.25 & 0.14 & 6.0 & 1.4 \\
\hline $1959+650$ & 0FGL J2000.2 + 6506 & 0.047 & 0.23 & $\ldots$ & 8 & 1.86 & 0.11 & 4.2 & 1.0 \\
\hline $2005-489$ & 0FGL J2009.4 - 4850 & 0.071 & 1.19 & $\cdots$ & 3 & 1.85 & 0.14 & 2.9 & 0.9 \\
\hline $2032+107$ & EGR J2032 + 1226 & 0.601 & 0.77 & $\ldots$ & 3 & 2.83 & 0.26 & 13.05 & 3.0 \\
\hline $2155-304$ & 0FGL J2158.8 - 3014 & 0.116 & 0.31 & $\ldots$ & 3 & 1.85 & 0.04 & 18.1 & 1.2 \\
\hline $2200+420$ & 0FGL J2202.4 + 4217 & 0.069 & 2.49 & $\ldots$ & 5 & 2.24 & 0.12 & 8.5 & 1.8 \\
\hline
\end{tabular}

